DOI: $10.15593 / 2224-9982 / 2017.48 .01$

УДК 621.762.222

\author{
И.Ю. Зубко, А.В. Зайцев
}

Пермский национальный исследовательский политехнический университет, Пермь, Россия

\author{
МОДЕЛИРОВАНИЕ ДВИЖЕНИЯ МЕЛЮЩИХ ТЕЛ \\ В ПРОЦЕССЕ МЕХАНИЧЕСКОГО ЛЕГИРОВАНИЯ
}

\begin{abstract}
Порошковые композиционные материалы, а также тонкие покрытия деталей, используемые в авиационной и ракетной технике, наследуют свойства и структуру порошков металлов и сплавов, приобретаемые в процессе механического легирования. Известно, что в зависимости от интенсивности передачи механической энергии в частицах порошка может формироваться различная внутренняя структура вплоть до нанокристаллической и аморфной. Задача выбора параметров работы устройств по производству порошка с целью получения необходимой микроструктуры частиц является нетривиальной и не всегда может быть решена экспериментальными методами. В работе строится математическая модель движения мелющих шаров при механическом легировании в шаровой планетарной мельнице. Рассматривается подход, в котором оценка энергетической эффективности шаровой планетарной мельницы проводится на основании анализа характера траектории отдельных мелющих шаров. В численных экспериментах определяются зависимости средних кинематических и динамических характеристик движения шаров от внешних параметров процесса. По найденным характеристикам делается оценка величины механической энергии, передаваемой частицам порошка. Получены варианты соотношения частот вращения барабана и чаш планетарной мельницы, позволяющие получить различную интенсивность передачи механической энергии частицам порошка.

Ключевые слова: порошковые материалы, механическое легирование, шаровая планетарная мельница,
\end{abstract} неупругие соударения мелющих шаров.

\author{
I.Yu. Zubko, A.V. Zaitsev
}

Perm National Research Polytechnic University, Perm, Russian Federation

\title{
SIMULATION OF MILLING BALLS MOTION WITHIN MECHANICAL ALLOYING PROCESS
}

\begin{abstract}
Powder composite materials and thin covers of details in aerospace engineering inherit properties and structure of metal and alloy powders formed within the mechanical alloying process. It is well known, that different values of intensity of the mechanical energy transition to the powder particles lead to different type of internal structure right up to nano-crystalline and even amorphous. Choosing the certain milling devices parameters with the aim of obtaining appropriate type of powder microstructure is nontrivial problem and could not be solved in the pure experimental way. In this paper the simulation of milling balls motion in the mechanical alloying process was considered. The considered approach gives estimation of energetic efficiency of the ball planetary mill on the base of the balls trajectory type analysis. The numerical experiments determine the dependences between averaged kinematic and dynamic characteristics of balls motion and outer parameters of the process. Based on the resulted characteristics, the authors estimated the energy, which is transferred to powder particles. The variants of milling frequencies ratio corresponding different values of intensity the energy transition from milling balls to powder particles are obtained.

Keywords: powder materials, mechanical alloying, milling balls motion in planetary mill, nonelastic collisions.
\end{abstract}

\section{Введение}

Процесс механического легирования используется для получения твердого раствора кристаллических материалов (обычно металлов и сплавов) в виде мелкодисперсного порошка в результате высокоэнергетического механического воздействия на частицы исходных порошков в планетарной мельнице [1]. Планетарная мельница представляет собой вращающийся вокруг своей оси барабан с чашами, вращающимися одновременно вокруг своих осей. Оси вращения барабана и чаш параллельны направлению силы тяжести. Чаши заполнены мелющими шарами, взаимодействующими друг с другом, с частицами порошка и стенками. За счет сложного движения чаш достигается ускорение шаров до сотен $g$. Процесс является существенно неравновесным и сопровождается диссипацией подводимой механической энергии на трех уровнях: 
макроскопическом - за счет сложного вихревого движения трущихся шаров, мезоскопическом - за счет повторяющихся процессов слипания и разрушения частиц порошка, микроспическом - за счет процессов диффузии и изменения дефектной структуры в частицах порошка.

Моделированию процесса механического легирования на различных масштабных уровнях посвящено немало исследований, включая как описание деформирования частиц порошка, так и движение мелющих тел. В серии работ [2-7] рассматривается модель упругопластического деформирования частиц порошка, в которой объединены решения задачи Герца о чисто упругом контакте и задачи Мориса-Кортни о чисто пластическом контакте тел. При этом переход от чисто упругого деформирования контактирующих частиц порошка к пластическому происходит при условии, что нормальное напряжение в частицах достигает значения их твердости. В частности, при исследовании изначально сферических частиц порошка получено, что в результате последовательности пластических деформаций в совокупности частиц начинают преобладать уплощенные частицы. Динамика движения самих шаров при этом рассматривалась в приближенной постановке. Авторы этой серии также провели моделирование движения единственного мелющего тела, имеющего форму прямого цилиндра с основанием в виде криволинейного треугольника $[8,9]$, в котором показали, что для ряда режимов применение такого тела оказывается более эффективным по сравнению с мелющими шарами. В работе [10] рассмотрено пять потенциальных моделей, описывающих распределение частиц порошка по размерам в ходе процесса механического легирования. Модели основываются на уравнениях Смолуховского для баланса явлений коагуляции-фрагментации и используют набор скоростей агрегирования и фрагментирования, зависящих от размеров частиц. Был разработан метод определения параметров модели по экспериментально определенному среднему размеру частиц. Распределение размеров, прогнозируемое этими моделями, хорошо соотносится с соответствующими экспериментальными результатами. Самые удачные модели определяют самое точное распределение частиц по размерам. Числовые значения некоторых скоростей агрегирования и фрагментирования качественно связаны со свойствами материалов из первоначальной загрузки. В обзоре $[11,12]$ дается критическая оценка трех важных направлений моделирования процесса механического легирования с помощью метода дискретных элементов: законов межчастичного взаимодействия, важности и выбора параметров контакта и, в итоге, реализации математической модели. Сложность задачи моделирования заключается в том, чтобы эффективно его использовать для усовершенствования промышленных процессов на практике. Существует несколько областей применения метода дискретных элементов (МДЭ) для анализа работы барабанных мельниц, в которых МДЭ является особенно эффективным. К ним относятся анализ движения массы для улучшения промышленных процессов, прогнозирования передаваемой мощности, проектирования конструкции мельницы и микромасштабное моделирование для расчета гранулометрического состава. В работах показано, что движение шихты в шаровой мельнице может быть рассчитано при помощи МДЭ. Для шаровой мельницы результаты моделирования были подтверждены сравнением с фотоснимками движения шихты. Кроме того, было показано, что энергия, передаваемая частицам порошка в шаровых мельницах, может быть спрогнозирована с точностью до $10 \%$. В работе [13] с помощью МДЭ изучен процесс дробления при механическом легировании в вибрационных мельницах с использованием разных размеров шаров, а также соотношений масс шаров и порошка (коэффициенты загрузки). Были использованы реагирующие материалы, для которых экзотермическая реакция запускалась механически после определенного времени дробления. Достижение нужной стадии дробления определялось экспериментально по температуре в чаше мельницы. Скачок температуры показывал время начала этой реакции. Было предложено выражение для вводимого параметра - дозы энергии, чтобы описать влияние различных параметров дробления на ход процесса. В первом приближении доза энергии, приводящая к механическому запуску реакции, оставалась неизменной для различных коэффициентов загрузки и, следовательно, могла использоваться для калибровки протекания процесса. Протекание процесса дробления было теоретически описано 
с помощью МДЭ. Было обнаружено, что теоретически введенная доза энергии, коррелирующая с экспериментальным аналогом, зависит от скорости диссипации энергии в прямых соударениях шаров. Представленный подход применим при моделировании промышленных устройств и для оптимизации механического легирования различных материалов. В статье [14] кратко описываются основы численного моделирования с помощью МДЭ для исследования некоторых особенностей динамики процесса шарового дробления. Были кратко представлены общие принципы подхода дискретных элементов с последующим обсуждением некоторых частных вычислительных вопросов, связанных с моделированием процессов в планетарных шаровых мельницах. Был представлен численный пример для демонстрации применимости разработанной процедуры. В работе [15] было исследовано влияние направления вращения чаши в шаровой планетарной мельнице и его скорости, а также отношения этой скорости к скорости вращения чаши на энергию соударений шаров, вычисляемую при моделировании на основе МДЭ. Измеренная энергия соударений оказывается большой, и структура частиц порошка переходит в аморфное состояние быстрее, когда чаша вращается в противоположном направлении по отношению к барабану. В обоих соотношениях направлений вращения энергия соударения растет с увеличением величины соотношения частот вращения на начальной стадии и затем падает вблизи критического соотношении скоростей, которое может быть вычислено из уравнения баланса сил инерции, действующих на шар. Самое высокое значение энергии соударения шаров при легировании может быть достигнуто вблизи критического соотношения скоростей. Следовательно, оно является ключевым условием для проектирования оптимального процесса механического легирования. К экзотическим подходам моделирования измельчения частиц порошка при механическом легировании можно отнести исследование [16], в котором была разработана искусственная нейронная сеть, предназначенная для моделирования влияния параметров легирования, включая время дробления, скорость дробления и соотношения масс шаров и порошка, на свойства нанокомпозитных порошков. Исследуемый наноструктурированный нанокомпозитный порошок был получен с помощью высокоэнергетической планетарной шаровой мельницы, и необходимые для обучения сети данные были подобраны по результатам экспериментов. Характеристики частиц были найдены при рентгенографическом исследовании, сканирующей и просвечивающей электронной микроскопии.

В данной работе строится численная модель, описывающая с помощью МДЭ процесс на макроуровне - движение шаров в мельнице. В качестве дискретных элементов рассматриваются шары, на которые действует сила инерции в системе отсчета, связанной с движущейся чашей шаровой планетарной мельницы. Шары взаимодействуют только контактным способом. Анализируются траектория движения шаров и сопоставление ее формы с уровнем энергии, которая может быть сообщена частицам порошка при различных частотах вращения барабана и чаши. Решение такой задачи позволит оценить уровень энергии, передаваемой на мезо- и микроскопический уровень, и определить средние характеристики воздействия на частицы порошка, попадающие между шарами, - входные данные для решения задачи на мезоуровне.

При механическом легировании системе шары - порошок сообщается кинетическая энергия, значительно превышающая потенциальную энергию системы, поэтому при описании движения шаров влиянием силы тяжести можно пренебречь. Взаимодействие элементов исследуемой системы включает повторяющиеся удары и трение шаров при непрерывном контакте. Последнее представляет собой движение с неголономными связями, и его описание для системы более чем двух тел сложно. При непрерывном контакте выбранного шара с несколькими шарами невозможно определить силы реакции между шарами - задача статически неопределима. При ударах же происходит мгновенный контакт тел, поэтому при моделировании ударов всегда можно выделить некоторую их последовательность, решая каждый раз задачу только для парного удара шаров. Далее для простоты будем учитывать лишь ударное взаимодействие шаров. Поскольку к рассматриваемой системе за счет сложного вращения непрерывно подводится механическая энергия, то проблему описания остановки шаров или перехода от режима 
движения в виде последовательности соударений к качению можно проигнорировать. Итак, рассматривается область в чаше планетарной мельницы, удаленная как от свободной поверхности, так и от дна. Процессы, идущие в этой части чаши в смежных слоях шаров, подобны, поэтому для упрощения учитывается только один слой шаров (центры масс шаров принадлежат одной плоскости).

\section{Соударение мелющих шаров}

В общем случае пространственного контакта твердых тел различают три вида трения: трение скольжения, трение качения и трение верчения. Два последних возникают как результат деформации тел вблизи точки контакта и представляют собой реактивные моменты связей в области контакта. Наибольшее значение при взаимодействии шаров имеет трение скольжения, поэтому будем пренебрегать трением качения и верчения. Коэффициент трения скольжения принимается постоянным, и при движении используется закон Амонтона-Кулона:

$$
F_{m}=f N,
$$

в покое же величина силы трения $F_{m}<f N$. Наличие между шарами порошка меняет характер трения. До превышения касательными усилиями предела текучести частиц порошка справедливо соотношение (1), где $f$ - коэффициент трения между частицами порошка и шарами, касательное усилие за пределом текучести постоянно и определяется последним (закон Зибеля). При механическом легировании мелющие шары испытывают сильные ударные воздействия, при которых нормальная компонента реакции способна достигать больших значений. В этих условиях порошок будет играть роль смазки, а его частицы будут деформироваться неупруго.

Все виды механического воздействий на $i$-й шар со стороны окружающих шаров и внешнего поля сил приводят к изменению его количества движения $\mathbf{Q}$ и момента количества движения К. Основные уравнения динамики твердого тела записываются относительно центра масс шара как

$$
\frac{d \mathbf{Q}_{i}}{d t}=\mathbf{R}_{i}^{(e)}, \frac{d \mathbf{K}_{i}}{d t}=\mathbf{M}_{i}^{(e)}
$$

где $\mathbf{R}_{i}^{(e)}$ - главный вектор; $\mathbf{M}_{i}^{(e)}$ - главный момент действующих на шар внешних сил. Уравнения (2) справедливы при непрерывном изменении воздействий на выбранный шар, при ударных же нагрузках используются другие уравнения. Удар является сообщением телу в некоторый момент времени $t_{0}$ конечного импульса I за бесконечно малый промежуток времени $\tau$, по истечении которого контакт между ударяющимися телами прекращается, $\mathbf{I}=\lim _{\tau \rightarrow 0} \int_{t_{0}}^{t_{0}+\tau} \mathbf{F} d t$. Изменение координат и ускорения тела за время удара не происходит, но скачком меняется скорость. Сила $\mathbf{F}$ при этом полагается бесконечно большой, для конечных сил $\mathbf{I}=0$. Основные уравнения импульсивного движения

$$
\Delta \mathbf{Q}_{i}=\mathbf{I}_{i}^{(e)}, \Delta \mathbf{K}_{i}=\mathbf{L}_{i}^{(e)},
$$

где $\mathbf{I}_{i}^{(e)}$ - результирующая внешних импульсов; $\mathbf{L}_{i}^{(e)}-$ результирующая моментов импульсов. В рассматриваемом случае однородных шаров все удары являются центральными, поэтому $\mathbf{L}_{i}^{(e)}$ содержит только моменты импульсов, передаваемых за счет трения между шарами. В правую часть уравнений (3) не входят непрерывно изменяющиеся конечные силы и моменты, стоящие в правой части (2). Уравнения (2) работают в промежутках между ударами, а в момент удара по уравнениям (3) определяется изменение скорости - начальные условия для (2) на следующем «безударном» отрезке времени. Если известны внешние импульсы, то из уравнений (3) по из- 
вестным доударным кинематическим характеристикам определяются все послеударные кинематические величины. При соударении же твердых тел величина ударного импульса заранее неизвестна и для решения уравнений (3) требуются дополнительные соотношения. Для этого рассмотрим две фазы контакта. В течение первой фазы происходит деформирование тел, причем скорость деформирования уменьшается до нуля, чем и определяется конец первой фазы. Деформация при этом максимальна. Затем начинается вторая фаза. Тела восстанавливают свою форму и в конце этой фазы соприкасаются в одной точке. Для абсолютно упругих тел энергия деформирования (запасенная в течение первой фазы) полностью возвращается при разгрузке (на второй фазе), т.е. независимо от модулей упругости скорости тел до и после удара будут совпадать по абсолютной величине (закон сохранения полной механической энергии при ударе абсолютно упругих тел). Для реальных тел абсолютная величина проекции на нормаль (общую для соударяющихся тел, проведенную в точке контакта в момент удара) не достигает после удара исходной величины. Часть механической энергии при этом теряется. Обозначим за $\mathbf{v}^{+}$ скорость поступательного движения центра масс тела после удара шаров 1 и 2 , а $\mathbf{v}^{-}-$скорость до удара. Для связи характеристик до и после удара принимается соотношение Ньютона

$$
\left(\mathbf{v}_{2}^{+}-\mathbf{v}_{1}^{+}\right) \cdot \mathbf{n}=-e\left(\mathbf{v}_{2}^{-}-\mathbf{v}_{1}^{-}\right) \cdot \mathbf{n},
$$

где $0 \leq e \leq 1$ - коэффициент восстановления; $\mathbf{n}-$ направление нормали к шарам в точке удара. При $e=1$ удар является абсолютно упругим, при $e=0$ происходит слипание тел. Полностью задача об определении послеударных кинематических характеристик при ударе с трением двух шаров решается методами теоретической механики с использованием уравнений (1), (3) и дополнительного соотношения (4). Например, в работе [17] приводится теория импульсивного движения и решение задачи об ударе произвольных тел без учета трения, в исследовании [18] учтено трение при описании удара материальной точки о неподвижное препятствие.

\section{Движение мелющих шаров при механическом легировании}

Для описания движения шаров перейдем в неинерциальную систему отсчета, связанную с подвижной чашей. Пусть $\omega_{1}$ и $\omega_{2}$ - постоянные угловые скорости вращения барабана и находящейся в нем чаши, $L$ - расстояние между осями вращения чаши и барабана, $\Phi-$ инерциальная система отсчета, $\Phi_{1}$ - неинерциальная система отсчета, вращающаяся вместе с барабаном, а $\Phi_{2}$ - с чашей относительно барабана. Перейдем сначала в систему $\Phi_{1}$, а затем в $\Phi_{2}$. Тогда ускорение а 2 материальной точки (или центра масс шара) относительно $\Phi_{2}$ связывается с ускорением а этой точки в инерциальной системе Ф как

$$
\begin{aligned}
& \underset{2}{\mathbf{a}}=\underset{2}{\mathbf{O}} \cdot \underset{1}{\mathbf{O}} \mathbf{\mathbf { a }}+\underset{2}{\mathbf{O}} \cdot\left(\underset{1}{\mathbf{\boldsymbol { \Omega }}}-\mathbf{\Omega}_{1}^{2}\right) \cdot\{L, 0\}+2\left[\underset{2}{\mathbf{O}} \cdot \underset{1}{\mathbf{\Omega}} \cdot \mathbf{O}^{T}+\underset{2}{\boldsymbol{\Omega}}\right] \cdot \underset{2}{\mathbf{V}}+
\end{aligned}
$$

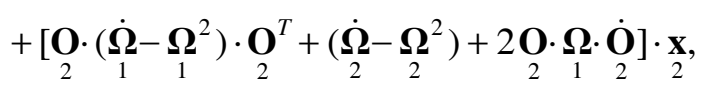

где $\underset{1}{\mathbf{O}}$ и $\underset{2}{\mathbf{O}}-$ тензоры поворотов Ф к $\Phi_{1}$ и $\Phi_{1}$ к $\Phi_{2}$ соответственно; $\underset{1}{\boldsymbol{\Omega}}$ и $\underset{2}{\boldsymbol{\Omega}}-$ тензоры спинов, построенные на $\underset{1}{\mathbf{O}}$ и $\underset{2}{\mathbf{O}} ; \underset{2}{\mathbf{x}}=\{x, y\}$ и $\underset{2}{\mathbf{v}}=\{\dot{x}, \dot{y}\}-$ радиус-вектор и скорость точки в системе $\Phi_{2}$. В инерциальной системе отсчета уравнение движения точки имеет вид $m \mathbf{a}=\mathbf{F}$, где $m$ - масса точки; $\mathbf{f}$ - результирующая действующих на нее сил. Учитывая, что $\mathbf{F}=\underset{1}{\mathbf{O}^{T}} \cdot \underset{2}{\mathbf{O}^{T}} \cdot \underset{2}{\mathbf{F}}$ и выражая из уравнения (5) ускорение а, получим закон движения в системе $\Phi_{2}$. В рассматриваемой постановке шары испытывают воздействия только при ударах, т.е. в промежутках между ударами $\underset{2}{\mathbf{F}}=0$, поэтому уравнения движения центра масс произвольного шара относительно $\Phi_{2}$ примут вид 


$$
\left\{\begin{array}{l}
\ddot{x}-2\left(\omega_{1}+\omega_{2}\right) \dot{y}-\left(\omega_{1}+\omega_{2}\right)^{2} x=L \omega_{1}^{2} \cos \left(\omega_{2} t\right), \\
\ddot{y}+2\left(\omega_{1}+\omega_{2}\right) \dot{x}-\left(\omega_{1}+\omega_{2}\right)^{2} y=-L \omega_{1}^{2} \sin \left(\omega_{2} t\right) .
\end{array}\right.
$$

Система (6) решается аналитически с заданными для некоторого шара начальными условиями $x(0)=x_{0}, y(0)=y_{0}, \dot{x}(0)=v_{x_{0}}, \dot{y}(0)=v_{y_{0}}:$

$$
\left\{\begin{array}{l}
x(t)=\left(A_{1}+A_{2} \omega_{2} t\right) \cos \left(\left(\omega_{1}+\omega_{2}\right) t\right)+ \\
+\left(A_{3}+A_{4} \omega_{2} t\right) \sin \left(\left(\omega_{1}+\omega_{2}\right) t\right)-L \cos \left(\omega_{2} t\right), \\
y(t)=\left(A_{3}+A_{4} \omega_{2} t\right) \cos \left(\left(\omega_{1}+\omega_{2}\right) t\right)- \\
-\left(A_{1}+A_{2} \omega_{2} t\right) \sin \left(\left(\omega_{1}+\omega_{2}\right) t\right)+L \sin \left(\omega_{2} t\right),
\end{array}\right.
$$

где

$$
\left\{\begin{array}{l}
A_{1}=L+x_{0}, A_{2}=v_{x_{0}} / \omega_{2}-\left(1+\omega_{1} / \omega_{2}\right) y_{0}, \\
A_{3}=y_{0}, A_{4}=v_{y_{0}} / \omega_{2}+\omega_{1} L / \omega_{2}+\left(1+\omega_{1} / \omega_{2}\right) x_{0} .
\end{array}\right.
$$

По системам (7) и (8) определяется время до соударения каждого шара со стенкой чаши и время до удара рассматриваемого шара с другими шарами. Первая задача сводится к определению ноля функции, выражающей расстояние от шара до стенки. Решение находится с помощью модифицированного метода Ньютона. Квадрат расстояния между двумя шарами выражается как квадратный трехчлен относительно $t$ (в этом выражении тригонометрических функций не остается), время до соударения между шарами определяется точно как наименьший положительный корень квадратного трехчлена.

Из связи скоростей вращения шара в $Ф$ и $\Phi_{2}$ получим

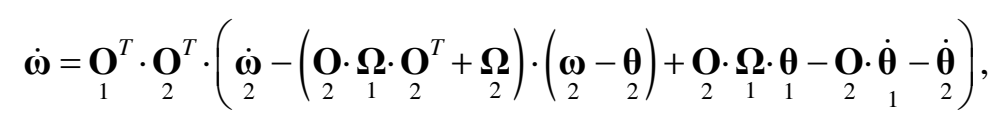

где $\underset{2}{\boldsymbol{\omega}}$ - скорость вращения шара относительно системы $\Phi_{2} ; \theta$ - аксиальный вектор спина $\Omega$. Моменты преобразуются как $\mathbf{M}=\underset{1}{\mathbf{O}^{T}} \cdot \underset{2}{\mathbf{O}^{T}} \cdot \underset{2}{\mathbf{M}}$. Учитывая, что на промежутках между ударами шар движется свободно, т.е. $\underset{2}{\mathbf{M}}=\mathbf{0}$, и что векторы угловых скоростей шаров, барабана и чаш направлены ортогонально рассматриваемой плоскости, получим уравнение вращательного движения относительно системы $\Phi_{2}$ :

$$
\underset{2}{\dot{\boldsymbol{\omega}}}=0
$$

Итак, в промежутках между ударами шары движутся с постоянными угловыми скоростями. В момент же удара за счет момента касательной составляющей ударного импульса угловые скорости вращения шаров изменяются мгновенно.

При численной реализации между шарами всегда оставляется зазор, т.е. все виды взаимодействия сводятся к ударам. За текущий шаг по времени на каждой итерации выбирается наименьший из следующих интервалов: 1) наименьшее время до удара некоторого шара о стенку; 2) наименьшее время до соударения пар шаров.

\section{Результаты и их обсуждение}

В расчетах брались параметры процесса, соответствующие реальным: скорость орбитального движения $\omega_{1}=700$ об/мин, скорость вращения чаши $\omega_{2}=1570$ об/мин, диаметр орбиты 20 см, чаши - 16 см, диаметр шаров - 1 см [1]. Для достижения наиболее плотного наполнения шарами рассматриваемого слоя при численном моделировании случайным образом разбра- 
сывались шары, затем делалось несколько расчетных циклов для «уплотнения» конфигурации и в освободившееся пространство снова случайным образом распределялись новые шары. Так было получено наполнение камеры до относительной доли шаров порядка 0,6 в плоском сечении, проходящем через центры шаров.

Эксперименты с одним шаром при $e=1$ (абсолютно упругие удары) и $f=0$ (трения нет) показали, что на поведение шара влияет сонаправленность скоростей вращения $\omega_{1}$ и $\omega_{2}$. При вращении в противоположные стороны происходит быстрое установление - траектория движения шара становится близкой к стационарной (рис. $1, a$ ), кинетическая энергия колеблется около неизменного уровня. Если же скорости направлены в одну сторону, то траектория движения шара заметает всю область (рис. 1,б), кинетическая энергия в этом случае возрастает. Если уменьшать коэффициент восстановления и не учитывать трение, то при $\omega_{1} \omega_{2}<0$ также происходит быстрое установление движения, энергия выходит на стационарный уровень, шар скользит вдоль стенки чаши. При $\omega_{1} \omega_{2}>0$ траектория заметает пространство, но энергия выходит на стационар (при уменьшении коэффициента восстановления шар также начинает скользить по стенке).

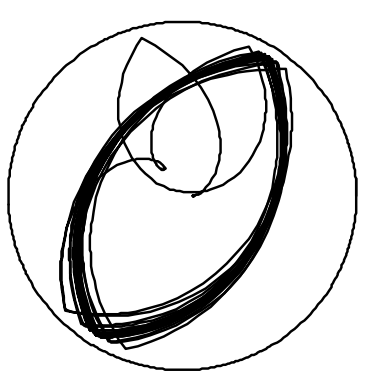

$a$

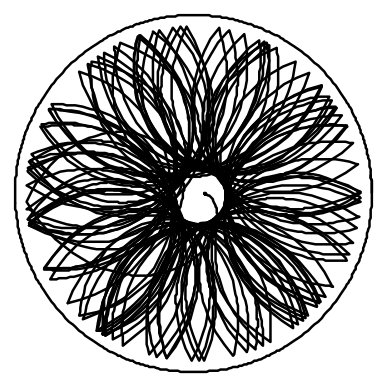

6

Рис. 1. Траектории центра масс одного шара при упругих ударах о стенку без учета трения: $a-\omega_{1} \omega_{2}<0 ; \sigma-\omega_{1} \omega_{2}>0$

В численном эксперименте с 50 шарами с абсолютно упругими ударами без трения вне зависимости от сонаправленности скоростей вращения энергия, приходящаяся на отдельный шар, выходит на стационар (рис. 2). В случае $\omega_{1} \omega_{2}>0$ этот средний уровень энергии выше, т.е. при совпадении направлений вращения барабана и чаши системе шаров передается большая энергия, движение шаров быстрее устанавливается.
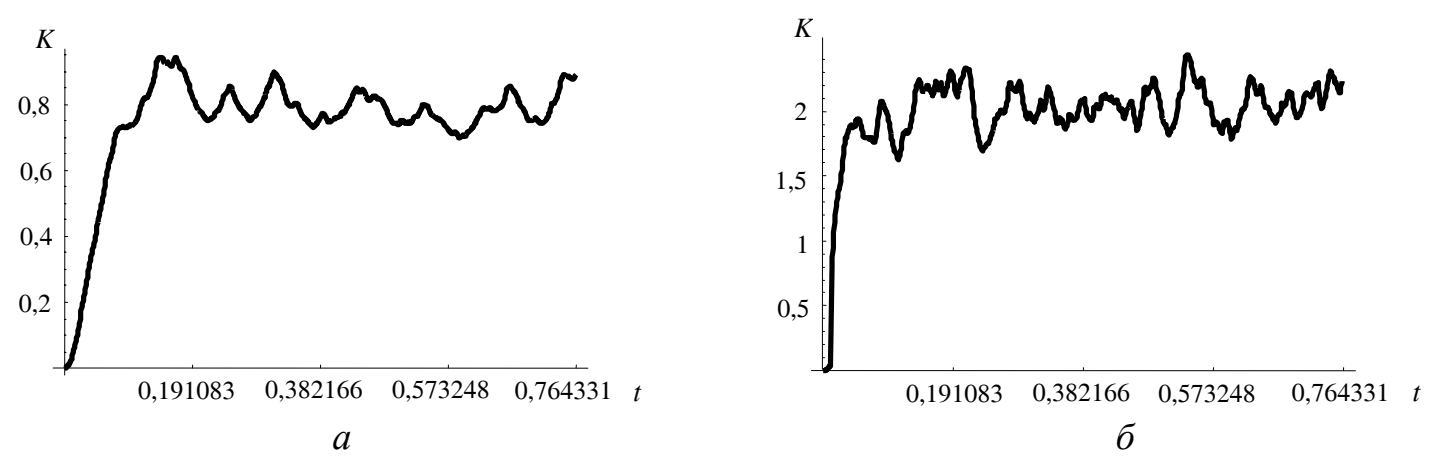

Рис. 2. Кинетическая энергия, приходящаяся на один шар. Опыт с 50 шарами: $a-\omega_{1} \omega_{2}<0 ; \sigma-\omega_{1} \omega_{2}>0$. Начальная конфигурация одинакова. Удары являются абсолютно упругими, трения нет. Время измеряется в секундах, метки на оси абсцисс кратны пяти оборотам чаши

Оценим далее величину механической энергии, подводимой к частицам порошка, в результате их взаимодействия с мелющими шарами. В модели диссипация энергии определяется коэффициентами восстановления и трения. Кинетическая энергия шара определяется как 


$$
K=\frac{1}{2} M v^{2}+\frac{1}{2} J \omega^{2}
$$

где $M$ - масса шара; $J$ - его момент инерции, $J=2 M R^{2} / 5$. Определим потерю энергии при ударе двух шаров $K^{-}-K^{+}$с учетом обмена импульсом при ударе с трением и некоторым коэффициентом восстановления $e$ :

$$
\Delta K^{*}=\frac{1}{2}\left[\frac{1-e^{2}}{2} M\left(w_{\text {отн }}^{-}\right)^{2}+2 T \tau_{\text {отн }}^{-}-7 \frac{T^{2}}{M}\right],
$$

где $w_{\text {отн }}^{-}$- величина нормальной составляющей относительной скорости шаров в момент удара; $\tau_{\text {отн }}^{-}-$величина касательной составляющей относительной скорости точек контакта при соударении двух шаров; $T$ - касательная составляющая ударного импульса, определяемого по $\tau_{\text {отн }}^{-}$, $e$ и $f$ в момент удара. Для оценки средней потери кинетической энергии при ударе шаров необходимо оценить средние характеристики $\left\langle w_{\text {отн }}^{-}\right\rangle,\left\langle T \tau_{\text {отн }}^{-}\right\rangle$и $\langle T\rangle$.

Построенная модель дает возможность оценивать эти величины в численных экспериментах и исследовать их зависимость от параметров процесса - частот вращений барабана и чаш, длительности перемалывания. По значению разности $\Delta K^{*}$ приближенно оценивается и доля энергии, сообщаемая отдельной частице.

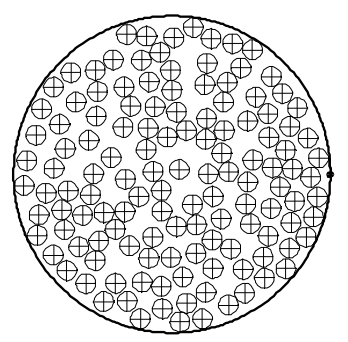

$a$

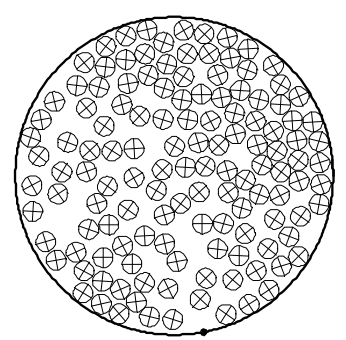

$\sigma$

Рис. 3. Конфигурации шаров в различные моменты времени расчета: $a$ - начальная конфигурация; $\sigma$ - конфигурация после пяти оборотов.

Точка на окружности показывает угол поворота чаши $\omega_{2} t$

На рис. 3 показаны две конфигурации системы шаров, полученные в модельных экспериментах с диссипацией. В расчетах определялись также осредненные величины нормальной и касательной составляющих ударного импульса при всех ударах, находились осредненные величины нормальной и касательной составляющих относительных скоростей. Эти характеристики нужны для оценки (9) и решения задачи о взаимодействии частиц порошка с мелющими телами и между собой как ее краевые условия. С течением времени количество ударов быстро возрастает и большая их часть происходит с проскальзыванием. Частицы порошка, попадающие при этом между шарами, должны деформироваться неупруго. При учете изменения свойств порошка коэффициент восстановления $е$ будет меняться, поэтому после решения задачи для частиц порошка необходимо уточнять решение и для шаров. В построенной модели не учитывается разогрев системы, наблюдаемый в реальном процессе. Однако даже в упрощенной постановке модель позволяет в зависимости от заданных параметров процесса получать оценки энергии, подводимой к отдельной частице, соответствующие экспериментам [1].

Работа выполнена при финансовой поддержке Российского фонда фундаментальных исследований (грант № 15-01-08678). 


\section{Библиографический список}

1. Проблемы порошкового материаловедения / под ред. В.Н. Анциферова. - Екатеринбург: УрО РАН, 2000. - Ч. І. - 252 с.

2. Шелехов Е.В., Свиридова Т.А. Компьютерное моделирование процесса механического сплавления в шаровых мельницах. Ч. І. Кинетико-статистическая геометрия изменений в обрабатываемом материале в процессе механического сплавления // Материаловедение. - 2007. - № 9. - С. 13-19.

3. Шелехов Е.В., Свиридова Т.А. Компьютерное моделирование процесса механического сплавления в шаровых мельницах. Ч. І. Кинетико-статистическая геометрия изменений в обрабатываемом материале в процессе механического сплавления (окончание I ч.) // Материаловедение. - 2007. - № 10. С. 13-22.

4. Шелехов Е.В., Свиридова Т.А. Компьютерное моделирование процесса механического сплавления в шаровых мельницах // Материаловедение. - 2007. - № 11. - С. 13-21.

5. Шелехов Е.В., Свиридова Т.А. Компьютерное моделирование процесса механического сплавления в шаровых мельницах // Материаловедение. - 2007. - № 12. - С. 10-15.

6. Шелехов Е.В., Свиридова Т.А. Компьютерное моделирование процесса механического сплавления в шаровых мельницах. Ч. III. Кинетика движения мелющих тел и расчет температуры мелющей среды // Материаловедение. - 2008. - № 2. - С. 10-22.

7. Шелехов Е.В., Свиридова Т.А. Компьютерное моделирование процесса механического сплавления в шаровых мельницах. Ч. ІІІ. Кинетика движения мелющих тел и расчет температуры мелющей среды (окончание III ч.) // Материаловедение. - 2008. - № 3. - С. 11-25.

8. Оценка величины интенсивности (энергонапряженности) механического легирования в планетарной мельнице с квазицилиндрическим мелющим телом / А.В. Тихомиров, А.А. Аксенов, Е.В. Шелехов, С.Д. Калошкин // Известия вузов. Цветная металлургия. - 2006. - № 3. - С. 51-59.

9. Шелехов Е.В., Свиридова Т.А. Компьютерное моделирование процесса механического сплавления. Ч. IV. Особенности модели для планетарного активатора с квазицилиндрическим мелющим телом // Материаловедение. - 2008. - № 4. - C. 16-24.

10. Harris J.R., Wattis J.A.D., Wood J.V. A comparison of different models for mechanical alloying // Acta Materialia. - 2001. - Vol. 49, № 19. - P. 3991-4003.

11. Mishra B.K. A review of computer simulation of tumbling mills by the discrete element method. Part I. Contact mechanics // Int. J. Miner. Process. - 2003. - № 71. - P. 73-93.

12. Mishra B.K. A review of computer simulation of tumbling mills by the discrete element method. Part II. Practical applications // Int. J. Miner. Process. - 2003. - № 71. - P. 95-112.

13. A study of mechanical alloying processes using reactive milling and discrete element modeling / T.S. Ward, W. Chen, M. Schoenitz, R.N. Dave, E.L. Dreizin // Acta Materialia. - 2005. - Vol. 53, № 10. P. 2909-2918.

14. Feng Y.T., Han K., Owen D.R.J. Discrete element simulation of the dynamics of high energy planetary ball milling processes // Materials Science and Engineering A. - 2004. - Vol. 375-377. - P. 815-819.

15. Effects of rotational direction and rotation-to-revolution speed ratio in planetary ball milling / H. Mio, J. Kano, F. Saito, K. Kaneko // Materials Science and Engineering A. - 2002. - Vol. 332. - P. 75-80.

16. Dashtbayazi M.R., Shokuhfar A., Simchi A. Artificial neural network modeling of mechanical alloying process for synthesizing of metal matrix nanocomposite powders // Materials Science and Engineering A. 2007. - Vol. 466, № 1-2. - P. 274-283.

17. Суслов Г.К. Теоретическая механика. - М.; Л.: Физматгиз, 1946. - 655 с.

18. Леви-Чивита Т., Амальди У. Курс теоретической механики. - М.: Изд-во иностр. лит-ры, 1951. - Т. 2. Ч. 2. $-556 \mathrm{c}$.

\section{References}

1. Antsiferov V.N. Problemy poroshkovogo materialovedeniya. Chast I [Problems of powder material science. Part I.]. Ekaterinburg, Ural Branch of RAS, 2000, 252 p.

2. Shelekhov E.V., Sviridova T.A. Kompyuternoe modelirovanie protsessa mekhanicheskogo splavleniya v sharovykh melnitsakh. Chast I. Kinetiko-statisticheskaya geometriya izmeneniy v obrabatyvaemom materiale v protsesse mekhanicheskogo splavleniya [Simulation of mechanical alloying in ball mills. Part I. Kineticstatistical geometry of transitions in processing material within mechanical alloying process]. Materialovedenie, 2007, no. 9, pp. 13-19. 
3. Shelekhov E.V., Sviridova T.A. Kompyuternoe modelirovanie protsessa mekhanicheskogo splavleniya v sharovykh melnitsakh. Chast I. Kinetiko-statisticheskaya geometriya izmeneniy v obrabatyvaemom materiale $\mathrm{v}$ protsesse mekhanicheskogo splavleniya (okonchanie $1 \mathrm{ch}$.) [Simulation of mechanical alloying in ball mills. Part I. Kinetic-statistical geometry of transitions in processing material within mechanical alloying process (continuation of part I)]. Materialovedenie, 2007, no. 10, pp. 13-22.

4. Shelehov E.V., Sviridova T.A. Kompyuternoe modelirovanie protsessa mekhanicheskogo splavleniya $\mathrm{v}$ sharovykh melnitsakh [Simulation of mechanical alloying in ball mills]. Materialovedenie, 2007, no. 11, pp. 13-21.

5. Shelehov E.V., Sviridova T.A. Kompyuternoe modelirovanie protsessa mekhanicheskogo splavleniya $\mathrm{v}$ sharovykh melnitsakh [Simulation of mechanical alloying in ball mills]. Materialovedenie, 2007, no. 12, pp. 10-15.

6. Shelehov E.V., Sviridova T.A. Kompyuternoe modelirovanie protsessa mekhanicheskogo splavleniya v sharovykh melnitsakh. Chast III. Kinetika dvizheniya melyushchikh tel i raschet temperatury melyushchey sredy [Simulation of mechanical alloying in ball mills. Part III. Kinetics of milling bodies motion and computation of milling mass temperature]. Materialovedenie, 2008, no. 2, pp. 10-22.

7. Shelehov E.V., Sviridova T.A. Kompyuternoe modelirovanie protsessa mekhanicheskogo splavleniya v sharovykh melnitsakh. Chast III. Kinetika dvizheniya melyushchikh tel i raschet temperatury melyushchey sredy (okonchanie III ch.) [Simulation of mechanical alloying in ball mills. Part III. Kinetics of milling bodies motion and computation of milling mass temperature (continuation of part III)]. Materialovedenie, 2008, no. 3, pp. 11-25.

8. Tihomirov A.V., Aksenov A.A., Shelekhov E.V., Kaloshkin S.D. Otsenka velichiny intensivnosti (energonapryazhennosti) mekhanicheskogo legirovaniya $\mathrm{v}$ planetarnoy melnitse $\mathrm{s}$ kvazitsilindricheskim melyushchim telom [Estimation of intensity value (energetic rating) for mechanical alloying in planetary mill with quasi-cylindrical milling body]. Izvestiya vysshih uchebnyh zavedenii. Cvetnaya metallurgiya, 2006, no. 3, pp. 51-59.

9. Shelehov E.V., Sviridova T.A. Kompyuternoe modelirovanie protsessa mekhanicheskogo splavleniya. Chast IV. Osobennosti modeli dlya planetarnogo aktivatora s kvazitsilindricheskim melyushchim telom [Simulation of mechanical alloying in ball mills. Part IV. Peculiarities of model for planetary activator with quasi-cylindrical milling body]. Materialovedenie, 2008, no. 4, pp. 16-24.

10. Harris J.R., Wattis J.A.D., Wood J.V. A comparison of different models for mechanical alloying. Acta Materialia, 2001, vol. 49, no. 19, pp. 3991-4003.

11. Mishra B.K. A review of computer simulation of tumbling mills by the discrete element method: Part I-contact mechanics. Int. J. Miner. Process, 2003, no. 71, pp. 73-93.

12. Mishra B.K. A review of computer simulation of tumbling mills by the discrete element method: Part II-Practical applications. Int. J. Miner. Process, 2003, no. 71, pp. 95-112.

13. Ward T.S., Chen W., Schoenitz M., Dave R.N., Dreizin E.L. A study of mechanical alloying processes using reactive milling and discrete element modeling. Acta Materialia, 2005, vol. 53, no. 10, pp. 29092918.

14. Feng Y.T., Han K., Owen D.R.J. Discrete element simulation of the dynamics of high energy planetary ball milling processes. Materials Science and Engineering A, 2004, vol. 375-377, pp. 815-819.

15. Mio H., Kano J., Saito F., Kaneko K. Effects of rotational direction and rotation-to-revolution speed ratio in planetary ball milling. Materials Science and Engineering A, 2002, vol. 332, pp. 75-80.

16. Dashtbayazi M.R., Shokuhfar A., Simchi A. Artificial neural network modeling of mechanical alloying process for synthesizing of metal matrix nanocomposite powders. Materials Science and Engineering: A, 2007, vol. 466, no. 1-2, pp. 274-283.

17. Suslov G.K. Teoreticheskaya mekhanika [Theoretical mechanics]. Moscow - Leningrad, Fizmatgiz, $1946,655 \mathrm{p}$.

18. Levi-Chivita T., Amaldi U. Kurs teoreticheskoy mekhaniki. Tom 2. Chast 2. [Course of theoretical mechanics. Vol. 2. Part 2]. Moscow, Izdatelstvo Inostrannoi literatury, 1951, 556 p.

\section{Об авторах}

Зубко Иван Юрьевич (Пермь, Россия) - кандидат физико-математических наук, доцент кафедры «Математическое моделирование систем и процессов» ФГБОУ ВО ПНИПУ (614990, г. Пермь, Комсомольский пр., 29, e-mail: zoubko@ list.ru). 
Зайцев Алексей Вячеславович (Пермь, Россия) - кандидат физико-математических наук, доцент кафедры «Механика композиционных материалов и конструкций» ФГБОУ ВО ПНИПУ (614990, г. Пермь, Комсомольский пр., д. 29, e-mail: zav@pstu.ru).

\section{About the authors}

Ivan Yu. Zubko (Perm, Russian Federation) - Ph. D. in Physical and Mathematical Sciences, Associate Professor, Department of Mathematical Modelling of Systems and Processes, Perm National Research Polytechnic University (29, Komsomolski av., Perm, 614990, Russian Federation, e-mail: zoubko@list.ru).

Aleksey V. Zaitsev (Perm, Russian Federation) - Ph. D. in Physical and Mathematical Sciences, Associate Professor, Department of Mechanics of Composite Materials and Structures, Perm National Research Polytechnic University (29, Komsomolski av., Perm, 614990, Russian Federation, e-mail: zav@pstu.ru).

Получено 07.11.2016 